\title{
Gastrointestinal autonomic neuropathy in diabetes: the unattended borderline between diabetology and gastroenterology
}

\author{
Péter Kempler $^{1}$ - Tamás Várkonyi ${ }^{2}$ - Anna E. Körei ${ }^{1}$ • Viktor J. Horváth ${ }^{1}$
}

Received: 15 October 2015 / Accepted: 5 November 2015 / Published online: 5 December 2015

(C) Springer-Verlag Berlin Heidelberg 2015

Keywords Diabetic enteropathy · Diabetic neuropathy . Gastrointestinal autonomic neuropathy · Gastroparesis . Postprandial hypoglycaemia

\section{Abbreviation \\ MMC Migrating motor complexes}

\begin{abstract}
'Autonomic dysfunction is often perceived as a black box of nebulous disorders, often not easily differentiated from variants of normality' [1]. On the one hand, this sentence is still absolutely valid: most consequences of autonomic neuropathy are mild and silent. However, on the other hand, at the tip of the iceberg, there are very severe manifestations of autonomic dysfunction, such as sudden death and gastroparesis.

In addition to cardiovascular autonomic dysfunction, gastrointestinal manifestations of autonomic neuropathy are common and relevant [2]. There are multiple levels of regulation in the gastrointestinal tract: the central, autonomic and enteric nervous systems and the interstitial cells of Cajal (mainly 'pacemaker cells'). The gut microbiota are also important. Moreover, many other factors probably affect gastrointestinal motility, including duration of diabetes, hyper- and hypoglycaemia, electrolyte disturbances, malnutrition and medications such as metformin, acarbose and glucagon-like
\end{abstract}

Péter Kempler

kempler.peter@med.semmelweis-univ.hu

1 1st Department of Medicine, Semmelweis University, Korányi S. utca 2/A, 1083 Budapest, Hungary

21 st Department of Medicine, University of Szeged, Szeged, Hungary peptide-1 analogues. The companion piece in this issue by Fernando Azpiroz and Carolina Malagelada discusses these aspects in more detail [3].

Data on the prevalence of gastrointestinal symptoms are inconsistent, but symptoms are generally very common, especially in women with diabetes. Differences in methodology may partly explain the conflicting results. Overall, the relationship between symptoms and altered gastrointestinal motility is relatively weak [4]. The frequency of symptoms is much higher, often $100 \%$, when data are reported by a gastroenterologist, but much lower if reported by a diabetologist. Fluctuating glucose levels and poor quality of life are the main consequences of impaired gastrointestinal function. Altered pharmacokinetics of drugs, insufficient or very variable absorption of important nutrients (potentially leading to malnutrition), impaired postprandial regulation of blood pressure, increased risk of gallstone formation and higher prevalence of gastrointestinal infections are other important consequences.

The paradox of dissociation of neuropathic gastrointestinal symptoms from objective evidence of neuropathic dysfunction is common. On the one hand, the absence of symptoms in patients with demonstrable markedly disordered gastric emptying may reflect an afferent nerve defect due to neuropathy. On the other hand, patients with symptoms have good evidence of visceral hypersensitivity, even though abnormalities of gastric emptying are not demonstrated on formal testing. Moreover, actual ambient glucose levels might influence the perception of gastrointestinal symptoms in central neuronal structures. These important facts contribute to the dissociation between subjective symptoms and objective motility findings.

Up to $50 \%$ of diabetic patients have some disorder of oesophagus motility. Reflux is frequently observed as a result of decreased tone of the lower oesophageal sphincter. The 
association between autonomic neuropathy and alterations of oesophageal motility is controversial.

\section{Diabetic gastroparesis}

An increased rate of stomach emptying may be present among patients with short diabetes duration without neuropathy [4]. Slower gastric emptying is present in the majority of diabetic patients with gastrointestinal autonomic neuropathy, as a consequence of reduced antral contractions, lack of migrating motor complexes (MMC) and antro-pyloro-duodenal incoordination [5]. Damage to the interstitial cells of Cajal also contributes to gastric dysrhythmias. The most characteristic symptoms are postprandial fullness, early satiety, nausea and vomiting, bloating and abdominal pain. Although a clear relationship between higher $\mathrm{HbA}_{1 \mathrm{c}}$ levels and the development and severity of gastroparesis has not been observed, poor glycaemic control is frequently the only manifestation of gastroparesis [6]. Postprandial hypoglycaemia, also known as gastric hypoglycaemia [7], may occur as a consequence of delayed food absorption in insulin-treated patients, usually among those with long-standing diabetes. Gastroparesis should be considered as the underlying mechanism in patients with otherwise unexplained periods of hypoglycaemia followed by hyperglycaemia, and should be excluded before establishing a diagnosis of brittle diabetes.

Acute myocardial infarction is frequently painless or silent in diabetic patients with autonomic neuropathy and is always associated with a rapid rise of blood glucose values [8, 9]. Acute hyperglycaemia leads to a rapid slowdown of gastric motility. Thus, an acute myocardial infarction should always be considered in a patient with rapid unexplained hyperglycaemia or ketoacidosis. As a consequence, acute myocardial infarction is particularly frequently associated with vomiting in diabetic patients.

Figure 1 summarises schematically the diagnostic approaches for gastroparesis and diabetic enteropathy. The symptoms of patients with upper and lower diabetesrelated gastrointestinal motility disorders may overlap [10], and the underlying mechanisms or cellular and histological alterations are also similar. Thus, if gastroparesis has been diagnosed, investigations of lower gastrointestinal motility disorders should be considered, and vice versa.

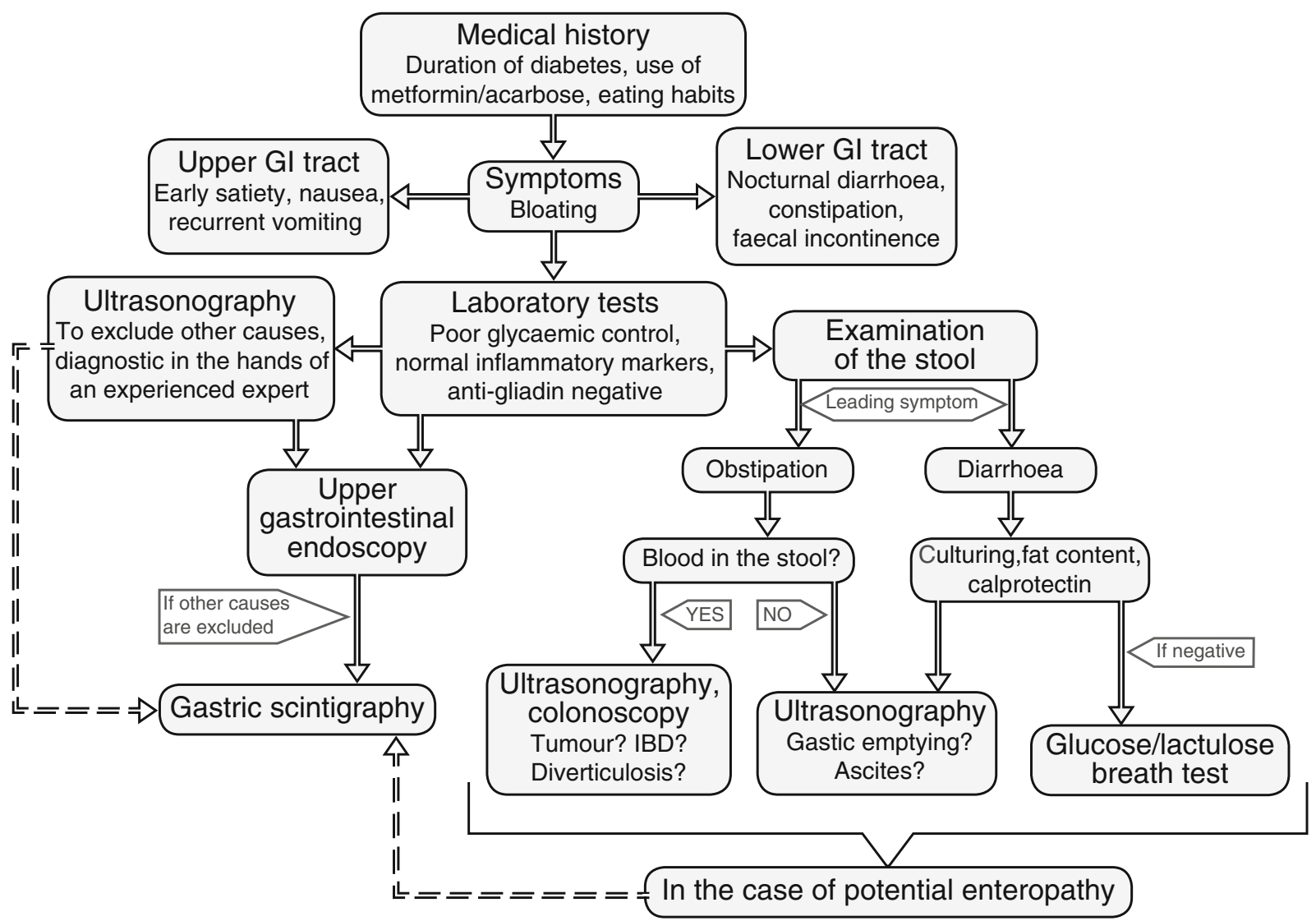

Fig. 1 Diagnostic approach for gastrointestinal motility disorders related to diabetic autonomic neuropathy. GI, gastrointestinal; IBD, inflammatory bowel disease 


\section{Diabetic enteropathy}

It is quite common for hyper- and hypofunction to occur simultaneously in several manifestations of diabetic neuropathy. Regarding sensory nerve function, hypoaesthesia is frequently preceded by hyperaesthesia [11]. Hyperfiltration can be observed in the early phase of diabetic nephropathy, while decreased glomerular filtration rate is the usual finding in later phases. In diabetic enteropathy, such a sequence of disorders is not usual. However, consequences of gut hypo- and hyperfunction are often observed simultaneously [12]. Constipation is the usual finding; however, paroxysmal nocturnal diarrhoea is also a characteristic finding of enteropathy. Obviously, other potential causes leading either to constipation or diarrhoea should be excluded. The lack of MMC phase 3 and antroduodenal incoordination are considered to be autonomic manifestations [13]. As a third leading symptom, faecal incontinence may occur. External anal sphincter weakness and anorectal incoordination are thought to be consequences of parasympathetic autonomic or central nervous dysfunction. A diagnostic approach for diabetic enteropathy is summarised in Fig. 1.

Several decades ago, gallbladder function was assessed by intravenous cholangiography, and the large, poorly contracting gallbladder with gallstones was the characteristic finding of autonomic involvement. Nowadays, cholangiography has been replaced by ultrasonography. We should be very careful when viewing ultrasound scans, as the large gallbladder with stones can be easily misinterpreted as a sign of acute inflammation.

The most common digestive complications of diabetes are impaired gastric motility, bowel dysfunctions and abdominal pain, and the therapeutic options for these complications are reviewed by Hans Törnblom in this issue [14].

In summary, the complexity of the neural regulation and the high number of reversible and irreversible pathogenetic factors modulating digestive and motility functions contribute to the diversity of the gastrointestinal manifestations in diabetic patients.

Funding Support by the Hungarian Diabetes Association is acknowledged.

Duality of interest The authors declare that there is no duality of interest associated with this manuscript.
Contribution statement All authors were responsible for drafting the article and revising it critically for important intellectual content. All authors approved the version to be published.

\section{References}

1. Narkiewicz K, Somers VK (1998) Chronic orthostatic intolerance: part of a spectrum of dysfunction in orthostatic cardiovascular homeostasis? Circulation 98:2105-2107

2. Kempler P, Ameranco G, Freeman R et al (2011) Gastrointestinal autonomic neuropathy, erectile-, bladder and sudomotor dysfunction in patients with diabetes mellitus: clinical impact, assessment, diagnosis and management. Diabetes Metab Res Rev 27:665-677

3. Azpiroz F, Malagelada C (2015) Diabetic neuropathy in the gut: pathogenesis and diagnosis. Diabetologia. doi:10.1007/s00125015-3831-1

4. Intagliata N, Koch KL (2007) Gastroparesis in type 2 diabetes mellitus: prevalence, etiology, diagnosis, and treatment. Curr Gastroenterol Rep 9:270-279

5. Izbéki F, Rosztóczy A, Várkonyi T, Wittmann T (2012) The clinical picture, diagnosis and therapy of gastrointestinal autonomic neuropathy. In: Kempler P, Várkonyi T (eds) Neuropathies. A global clinical guide. Zafír Press, Budapest, pp 131-150

6. Horváth V, Izbéki F, Cs L, Kempler P, Várkonyi T (2014) Diabetic gastroparesis: functional/morphologic background, diagnosis and treatment options. Curr Diab Rep 14:527

7. Horowitz M, Jones KL, Rayner CK, Read NW (2006) 'Gastric' hypoglycaemia: an important concept in diabetes management. Neurogastroenterol Motil 18:405-407

8. Wackers FJ, Young LH, Inzucchi SE et al (2004) Detection of silent myocardial ischemia in asymptomatic diabetic subjects: the DIAD study. Diabetes Care 27:1954-1961

9. Spallone V, Ziegler D, Freeman R et al (2011) Cardiovascular autonomic neuropathy in diabetes: clinical impact, assessment, diagnosis, and management. Diabetes Metab Res Rev 27:639-653

10. Phillips LK, Rayner CK, Jones KL, Horowitz M (2006) An update on autonomic neuropathy affecting the gastrointestinal tract. Curr Diab Rep 6:417-423

11. Barkai L, Kempler P, Vámosi I, Lukács K, Marton A, Keresztes K (1998) Peripheral sensory nerve dysfunction in children and adolescents with type 1 diabetes mellitus. Diabetic Med 15:228-233

12. Horváth V, Putz Z, Izbéki F et al (2015) Diabetes-related dysfunction of the small intestine and the colon: focus on motility. Curr Diab Rep 15:94. doi:10.1007/s11892-015-0672-8

13. Deloose E, Janssen P, Depoortere I, Tack J (2012) The migrating motor complex: control mechanisms and its role in health and disease. Nat Rev Gastroenterol Hepatol 9:271-285

14. Törnblom H (2015) Treatment of gastrointestinal autonomic neuropathy. Diabetologia. doi:10.1007/s00125-015-3828-9 